\title{
Granulicatella adiacens
}

National Cancer Institute

\section{Source}

National Cancer Institute. Granulicatella adiacens. NCI Thesaurus. Code C86426.

A species of facultatively anaerobic, Gram positive, rod or cocci shaped bacteria assigned

to the phylum Firmicutes. This species is nonmotile, non spore forming, catalase and oxidase negative, and produces pyrrolidonyl arylamidase. G. adiancens is a pathogen which can be isolated from the throat, blood or urine and most commonly causes endocarditis. 